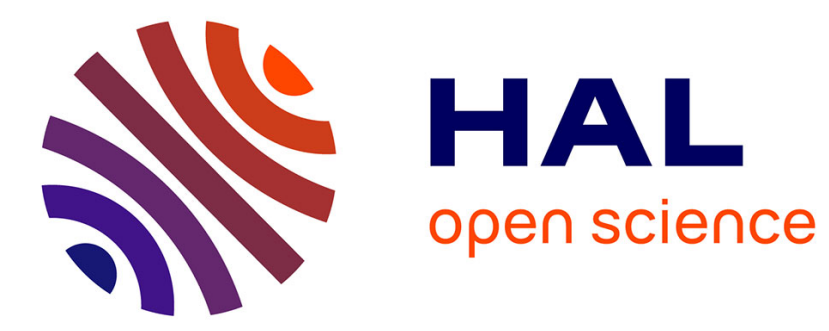

\title{
Strong iISS for a class of systems under saturated feedback
}

\author{
Rémi Azouit, Antoine Chaillet, Yacine Chitour, Luca Greco
}

\section{To cite this version:}

Rémi Azouit, Antoine Chaillet, Yacine Chitour, Luca Greco. Strong iISS for a class of systems under saturated feedback. Automatica, 2016, 71, pp.272 - 280. 10.1016/j.automatica.2016.04.023 . hal01098374

\section{HAL Id: hal-01098374 https://hal.science/hal-01098374}

Submitted on 24 Dec 2014

HAL is a multi-disciplinary open access archive for the deposit and dissemination of scientific research documents, whether they are published or not. The documents may come from teaching and research institutions in France or abroad, or from public or private research centers.
L'archive ouverte pluridisciplinaire HAL, est destinée au dépôt et à la diffusion de documents scientifiques de niveau recherche, publiés ou non, émanant des établissements d'enseignement et de recherche français ou étrangers, des laboratoires publics ou privés. 


\title{
Strong iISS for a class of systems under saturated feedback $^{1}$
}

\author{
Rémi Azouit ${ }^{\mathrm{a}, \mathrm{b}}$, Antoine Chaillet ${ }^{\mathrm{a}}$, Yacine Chitour ${ }^{\mathrm{a}}$, Luca Greco ${ }^{\mathrm{a}}$ \\ ${ }^{\mathrm{a}}$ Univ. Paris Sud 11 - L2S - Supélec. 3, rue Joliot-Curie, 91192, Gif-sur-Yvette, France. \\ ${ }^{\mathrm{b}}$ ENS Cachan - Master ATSI, same address.
}

\begin{abstract}
This paper proposes sufficient conditions under which a system can be made Strongly iISS in the presence of actuator saturation. This property was recently proposed as a compromise between the strength of ISS and the generality of iISS. It ensures in particular that solutions are bounded provided that the disturbance magnitude is below a certain threshold, and that they tend to the origin in response to any vanishing disturbance. We show that, under a growth rate condition, the bounded feedback law proposed by Lin and Sontag for the stabilization of the disturbance-free system based on the knowledge of a control Lyapunov function, ensures Strong iISS in the presence of perturbations. We illustrate our findings on the angular velocity control of a spacecraft with limited-power thrusters. In the specific case of linear time-invariant systems with neutrally stable internal dynamics, we provide a simple static state-feedback that ensures Strong iISS in presence of actuator saturations. This second result is illustrated by the robust stabilization of the harmonic oscillator. In both cases, we provide an estimate of the maximum disturbance amplitude that can be tolerated without compromising solutions' boundedness.
\end{abstract}

Key words: Stability and robustness of nonlinear systems, input-to-state stability, saturated feedback

\section{Introduction}

It is well known that a necessary and sufficient condition for the stabilizability of a linear time-invariant (LTI) plant by saturated feedback is that the internal dynamics has no pole with positive real part (Sontag, 1998b). Several works in the literature have proposed bounded stabilizing feedback for particular classes of systems whose internal dynamics exhibits no exponential instability. For LTI systems having no eigenvalues with positive real parts, it has been shown in Sontag and Sussmann (1990) that global stabilization by bounded output feedback can be achieved if and only if the system is both detectable and stabilizable. For neutrally stable systems (meaning LTI systems whose internal dynamics exhibits no unbounded solutions), it is also known that stabilization can be achieved using a saturated linear static feedback.

Nonetheless some classes of systems, although having no poles with positive real parts, cannot be stabilized by saturated linear static state-feedback; this class includes chains of three or more integrators (Fuller, 1969; Sussmann and Yang, 1991). Nested saturations Teel (1992); Sussmann et al. (1993) have been proposed to stabilize such systems. Stabilization by bounded control has also proved useful for nonlinear dynamics, especially in the context of systems in feedforward form (Mazenc and Praly, 1996; Teel, 1996) or by relying on the so-called "universal constructions" (Lin and Sontag, 1991).

Email addresses: remi.azouit@lss.supelec.fr (Rémi Azouit), antoine.chaillet@supelec.fr (Antoine Chaillet), yacine.chitour@lss.supelec.fr (Yacine Chitour), luca.greco@lss.supelec.fr (Luca Greco).

1 This work has received funding from the European Union Seventh Framework Programme [FP7/2007-2013] under grant agreement n257462 HYCON2 Network of excellence, and from the iCODE institute, research project of the IDEX Paris-Saclay. 
Beyond stabilization, it is often desirable to ensure some robustness properties in order to cope, for instance, with parameter uncertainty, measurement noise or exogenous disturbances. $L_{p}$-stabilization with respect to disturbances acting inside the saturation was achieved in Saberi et al. (2000) based on the low-and-high gain control law introduced in Megretski (1996). This robust stabilization has been extended to disturbances acting outside the saturation in Wang et al. (2012) for chains of integrators under matching conditions. Also, explicit estimates of $L_{p}$ input/output gains have been obtained for neutrally stable systems based on a saturated linear static feedback (Liu et al., 1996). Another natural candidate for the evaluation of robustness to exogenous inputs is the framework of input-to-state stability (ISS, Sontag $(1989 a, 2006)$ ) and its weaker variant integral ISS (iISS, Sontag (1998a)). In Angeli et al. (2005), a saturated linear state-feedback is proposed that ensures ISS with respect to sufficiently small disturbances despite parameter uncertainty for systems of dimension smaller than or equal to three, as well as feedforward systems. ISS of neutrally stable systems with respect to disturbances acting outside the saturation have been proposed in Arcak and Teel (2002) under matching conditions. Other approaches guarantee ISS and iISS with bounded control to nonlinear systems based on the aforementioned "universal constructions" (Liberzon, 2002).

Among other robustness features, ISS ensures a bounded response to any bounded disturbance. Intuitively, one may expect that bounded controls fail in general at guaranteeing the solutions' boundedness if the disturbance acts outside the saturation with a too large amplitude (unless matching conditions between the saturated actuator and the disturbance are imposed: seee.g. Arcak and Teel (2002); Wang et al. (2012)). At first sight, for these systems, nothing more than ISS with respect to small inputs can be established, thus providing no information on the system's behavior for larger inputs. In this note, we provide sufficient conditions under which a more interesting property, namely Strong iISS, can be achieved by saturated feedback. This property, introduced in Chaillet et al. (2014a), not only guarantees ISS with respect to small inputs but also iISS. In particular, it ensures a bounded response to any disturbance whose amplitude is below a given threshold, but also the existence of solutions at all times even for disturbances above that threshold. It also guarantees that the state converges to zero in response to any vanishing disturbance, and is known to be preserved under cascade interconnection (Chaillet et al., 2014b).

In this paper, we identify two classes of systems for which Strong iISS can be achieved by saturating feedback. The first one is made of input-affine systems that can be internally stabilized by the "universal construction" of Lin and Sontag (1991) and for which a specific growth rate condition holds. The second one is the class of neutrally stable LTI systems, for which a saturated linear state feedback is shown to ensure Strong iISS. In both cases, the considered disturbances act outside the saturation, and no matching condition between the actuation and the disturbances is assumed. We start by formulating the problem and motivating it through an example (Section 2). Then, in Section 3, we provide a sufficient condition under which Strong iISS is achieved by saturated feedback and provide growth rate conditions under which the "universal construction" originally proposed to ensure global asymptotic stability of the disturbance-free system also guarantees Strong iISS in presence of perturbations. We provide an explicit estimate of the maximal disturbance amplitude that can be tolerated without compromising solutions' boundedness. We illustrate our findings through the stabilization of the Euler equations of a rotating spacecraft. Finally, focusing on LTI systems whose internal dynamics is neutrally stable, we propose in Section 4 a simple linear state feedback that ensures Strong iISS to additive disturbance despite actuator saturation. Here also, we provide an explicit estimate of the maximum tolerable disturbance amplitude and compare it to numerical observations in an example. Proofs are provided in Section 5. Conclusive remarks and perspectives are given in Section 6.

Notation. For a nondecreasing continuous function $\gamma: \mathbb{R}_{\geq 0} \rightarrow \mathbb{R}_{\geq 0}, \gamma(\infty) \in \mathbb{R}_{\geq 0} \cup\{\infty\}$ denotes the quantity $\lim _{s \rightarrow+\infty} \gamma(s)$. A function $\alpha: \mathbb{R}_{\geq 0} \rightarrow \mathbb{R}_{\geq 0}$ is of class $\mathcal{P} \mathcal{D}$ if it is continuous and positive definite. It is of class $\mathcal{K}$ if, in addition, it is increasing. It is of class $\mathcal{K}_{\infty}$ if it is of class $\mathcal{K}$ and $\alpha(\infty)=\infty$. $\beta: \mathbb{R}_{>0} \times \mathbb{R}_{>0} \rightarrow \mathbb{R}_{>0}$ belongs to class $\mathcal{K} \mathcal{L}$ if, given any fixed $t \geq 0, \beta(\cdot, t) \in \mathcal{K}$ and, given any fixed $s \geq 0, \beta(s, \cdot)$ is continuous, nonincreasing and tends to zero as its argument tends to infinity. Given $x \in \mathbb{R}^{n},|x|$ denotes its Euclidean norm. Given a positive integer $p, \mathcal{U}^{p}$ denotes the set of all measurable locally essentially bounded functions $d: \mathbb{R}_{>0} \rightarrow \mathbb{R}^{p}$. For a given $d \in \mathcal{U}^{p}$, $\|d\|:=\operatorname{ess} \sup _{t \geq 0}|d(t)|$. Given a constant $R>0$, we let $\mathcal{U}_{<R}^{p}$ denote the set $\left\{d \in \mathcal{U}^{p}:\|d\|<R\right\}$. sat $: \mathbb{R}^{n} \rightarrow \mathbb{R}^{n}$ is the vector saturation function defined as $\operatorname{sat}(x)=\left(\operatorname{sat}^{0}\left(x_{1}\right), \ldots, \operatorname{sat}^{0}\left(x_{n}\right)\right)^{T}$, where $\operatorname{sat}^{0}(s):=\min \{1 ;|s|\} \operatorname{sign}(s)$ for each $s \in \mathbb{R}$. A function $V: \mathbb{R}^{n} \rightarrow \mathbb{R}_{\geq 0}$ is called a storage function if it is continuously differentiable and satisfies $V(0)=0$ and $V(x)>0$ for all $x \neq 0$. A storage function is said to be proper if, in addition, $\lim _{|x| \rightarrow \infty} V(x)=\infty$. Given a storage function $V$ and a vector field $f: \mathbb{R}^{n} \rightarrow \mathbb{R}^{n}, L_{f} V(x):=\frac{\partial V(x)}{\partial x} f(x)$. 


\section{Problem statement}

Consider a nonlinear system of the form $\dot{x}=f(x, u, d)$, where $x \in \mathbb{R}^{n}$ is the state, $u \in \mathbb{R}^{m}$ is the control input, $d \in \mathbb{R}^{p}$ the exogenous disturbance and $f: \mathbb{R}^{n} \times \mathbb{R}^{m} \times \mathbb{R}^{p} \rightarrow \mathbb{R}^{n}$ denotes a locally Lipschitz function satisfying $f(0,0,0)=0$. If the system is stabilized through a static state feedback $u=k(x)$, the system takes the form

$$
\dot{x}=\tilde{f}(x, d),
$$

where $\tilde{f}(x, d):=f(x, k(x), d)$. Given $x_{0} \in \mathbb{R}^{n}$ and an input signal $d \in \mathcal{U}^{m}$, the solution of (1) starting at $x_{0}$ at time $t=0$ is referred to as $x\left(\cdot, x_{0}, d\right)$ (or simply $\left.x(\cdot)\right)$ on the time domain where it is defined.

Assume that the state feedback $k(x)$ is nominally designed to ensure input-to-state stability (ISS, Sontag (1989a, 2006)) of the closed-loop system (1). Such a control law may be designed using existing techniques from the literature, such as Sontag (1990); Krstic and Li (1998); Teel and Praly (2000); Liberzon et al. (2002); Malisoff et al. (2004). Then, a natural question is to know what kind of robustness can be guaranteed to (1) in the presence of actuator saturation. Intuitively, we can expect that the applied control input $u=\operatorname{sat}(k(x))$ fails at guaranteeing a bounded state in response to disturbance of too large magnitude, thus compromising ISS. Nonetheless a weaker robustness property, namely iISS (Sontag, 1998a), can reasonably be expected.

Definition 1 (iISS) The system (1) is said to be integral input-to-state stable if there exist a class $\mathcal{K} \mathcal{L}$ function $\beta$ and class $\mathcal{K}_{\infty}$ functions $\mu_{1}, \mu_{2}$ such that, for all $x_{0} \in \mathbb{R}^{n}$ and all $d \in \mathcal{U}^{p}$, its solution satisfies, for all $t \geq 0$,

$$
\left|x\left(t ; x_{0}, d\right)\right| \leq \beta\left(\left|x_{0}\right|, t\right)+\mu_{1}\left(\int_{0}^{t} \mu_{2}(|d(s)|) d s\right) .
$$

Unfortunately, even if iISS systems prove robust with respect to classes of inputs with finite energy (in particular, if the energy $\int_{0}^{\infty} \mu_{2}(|d(s)|) d s$ is finite, then the state converges to the origin), solutions can run unbounded in the presence of arbitrary small and even vanishing inputs (Chaillet et al., 2014a). Generically, we may expect a bounded state property at most for disturbances whose amplitudes are below a specific threshold. That is, we could consider systems which are ISS with respect to small inputs.

Definition 2 (ISS wrt small inputs) The system (1) is said to be input-to-state stable with respect to small inputs if there exist a constant $R>0$, a class $\mathcal{K} \mathcal{L}$ function $\beta$ and a class $\mathcal{K}_{\infty}$ function $\mu$ such that, for all $x_{0} \in \mathbb{R}^{n}$ and all $d \in \mathcal{U}^{p}$, its solution satisfies

$$
\|d\|<R \Rightarrow\left|x\left(t ; x_{0}, d\right)\right| \leq \beta\left(\left|x_{0}\right|, t\right)+\mu(\|d\|), \forall t \geq 0 .
$$

In the case when $R=+\infty$, we recover the classical definition of ISS (Sontag, 1989a, 2006). However, given a finite $R$, no guarantee on the behavior of the system can be given when the disturbance magnitude overpasses the threshold $R$. The very solution of the system may fail to exist if $\|d\| \geq R$. Hence, a good candidate to evaluate the robustness to exogenous disturbances of systems with saturated feedback seems to be the Strong iISS, recently introduced in Chaillet et al. (2014a).

Definition 3 (Strong iISS) The system (1) is said to be Strongly iISS if it is both ISS wrt small inputs and iISS. In other words, there exist $R>0, \beta \in \mathcal{K} \mathcal{L}$ and $\mu_{1}, \mu_{2}, \mu \in \mathcal{K}_{\infty}$ such that, for all $d \in \mathcal{U}^{p}$, all $x_{0} \in \mathbb{R}^{n}$ and all $t \geq 0$, its solution satisfies the two properties:

$$
\begin{gathered}
|x(t)| \leq \beta\left(\left|x_{0}\right|, t\right)+\mu_{1}\left(\int_{0}^{t} \mu_{2}(|d(s)|) d s\right) \\
\|d\|<R \Rightarrow|x(t)| \leq \beta\left(\left|x_{0}\right|, t\right)+\mu(\|d\|) .
\end{gathered}
$$

The constant $R$ is then called an input threshold. 
Nonetheless, the link between Strong iISS and systems with saturated control is not straightforward. For instance, elementary considerations convince that not every nominal ISS-stabilizing feedback ensures Strong iISS once saturated. Indeed, saturated feedback may be insufficient to compensate for unbounded sources of instability, thus compromising even the internal stability of the plant. Since iISS, and consequently Strong iISS, imply global asymptotic stability in the absence of disturbance (this property will be called 0-GAS in the rest of the article), only systems that can be globally stabilized by saturated feedback can be expected to yield Strong iISS in the presence of actuator saturation. In other words, Strong iISS stabilization through saturated feedback faces all the challenges of global asymptotic stabilization by bounded control. In particular, for LTI systems, necessary requirements include stabilizability and the absence of eigenvalues with positive real parts (Sussmann et al., 1994). Based on these observations, a more reasonable hypothesis would be that any ISS-stabilizing nominal feedback that ensures 0-GAS when saturated, also ensures Strong iISS. The following example shows that this conjecture is not true in general.

Example 1 Consider the scalar system

$$
\dot{x}=x^{2} u+x^{3} d .
$$

The nominal feedback law $u=k(x)=-x^{5}$ makes the closed-loop system ISS. Indeed, the total derivative of the storage function $V(x)=x^{2} / 2$ reads

$$
\dot{V}(x)=-x^{8}+x^{4} d \leq-x^{8}+x^{4}|d| \leq-\frac{x^{8}}{2}+\frac{d^{2}}{2}
$$

which guarantees ISS by its classical Lyapunov characterization (Sontag and Wang, 1995). In the presence of actuator saturation, the closed-loop system becomes $\dot{x}=-x^{2}$ sat $^{0}\left(x^{5}\right)+x^{3} d$. Clearly, this system remains 0-GAS. Nonetheless it can be seen that, given any constant input $d^{*}>0$, any solution starting from $x_{0} \geq \max \left\{1 ; 2 / d^{*}\right\}$ grows unbounded, and even presents finite escape times).

The above example highlights the necessity to conduct a more careful study on how the Strong iISS property may be ensured by saturated feedback. This is the purpose of this paper.

\section{Nonlinear systems: "universal" construction}

We start by focusing on nonlinear input-affine systems:

$$
\dot{x}=f(x)+g(x) u+h(x) d
$$

where $x \in \mathbb{R}^{n}$ is the state, $u \in \mathbb{R}^{m}$ is the control input, and $d \in \mathbb{R}^{p}$ is the perturbation. The functions $f: \mathbb{R}^{n} \rightarrow \mathbb{R}^{n}$, $g: \mathbb{R}^{n} \rightarrow \mathbb{R}^{n \times m}$ and $h: \mathbb{R}^{n} \rightarrow \mathbb{R}^{n \times p}$ are assumed locally Lipschitz and zero at zero. In the presence of actuator saturation, the system reads

$$
\dot{x}=f(x)+g(x) \operatorname{sat}(u)+h(x) d .
$$

We stress that, if each control entries $u_{i}$ saturates at a value $\bar{u}_{i} \neq 1$, considering $\tilde{u}:=\left(u_{1} / \bar{u}_{1}, \ldots, u_{n} / \bar{u}_{n}\right)^{T}$ as the new control input allows to fit the framework (6).

\subsection{Sufficient conditions for Strong iISS}

We start by stating an analysis result, which provides sufficient conditions under which a generic control guarantees Strong iISS to (6). This result will serve as a basis to design saturated control laws in the next subsections. This first result relies on the following two assumptions, involving a proper storage function $V: \mathbb{R}^{n} \rightarrow \mathbb{R}_{\geq 0}$ and a locally Lispschitz state feedback $k: \mathbb{R}^{n} \rightarrow \mathbb{R}^{m}$.

Assumption 1 There exists a class $\mathcal{K}$ function $\gamma$ such that, for all $x \neq 0$,

$$
\begin{gathered}
L_{f} V(x)+L_{g} V(x) k(x)<0 \\
L_{h} V \neq 0 \Rightarrow\left|\frac{L_{f} V+L_{g} V k(x)}{L_{h} V}\right| \geq \gamma(|x|) .
\end{gathered}
$$


Assumption 1 contains two ingredients: (7) guarantees the 0-GAS of (5) in closed loop with $u=k(x)$, and, as we will see in the proof of Theorem 1 below, the combination of (7) and (8) ensures ISS wrt small inputs.

Assumption 2 It holds that

$$
\limsup _{|x| \rightarrow \infty} \frac{\left|L_{h} V(x)\right|}{1+V(x)}<+\infty
$$

Assumption 2 essentially guarantees that the perturbation does not yield finite escape times that a saturated feedback would not be able to tackle. This can be seen by observing that, by the continuity of the function $x \mapsto \frac{\left|L_{h} V(x)\right|}{1+V(x)},(9)$ ensures that

$$
\frac{\left|L_{h} V(x)\right|}{1+V(x)} \leq K, \quad \forall x \in \mathbb{R}^{n},
$$

for some positive constant $K$. Consequently, all the terms induced by the perturbation term in the total derivative of $V$ is at most linear in $V(x)$. This, combined with Assumption 1, constitutes a sufficient condition for forward completeness (Angeli and Sontag, 1999).

Based on these assumptions, we can state the following.

Lemma 1 Assume that there exists a proper storage function $V: \mathbb{R}^{n} \rightarrow \mathbb{R}_{\geq 0}$, a function $\gamma \in \mathcal{K}$, and a locally Lipschitz state feedback $k: \mathbb{R}^{n} \rightarrow \mathbb{R}_{\geq 0}$ satisfying Assumptions 1 and 2. Then the control law $u=k(x)$ makes the system (5) Strongly iISS with input threshold $R=\gamma(\infty)$.

The proof is rather straightforward, but is provided in Section 5.1 for the sake of completeness. It is worth stressing that Lemma 1 would not hold if either Assumption 1 or 2 was removed. Assumption 2 alone does not provide any information on the system in the absence of exogenous perturbations. Moreover, it can easily be seen that the system (4) of Example 1 satisfies Assumption 1, but fails at fulfilling Assumption 2. It results as a non Strongly iISS system, since the system is not forward complete in the presence of arbitrary small (and even vanishing) perturbations.

\subsection{Strong iISS by "universal" construction}

Although Lemma 1 gives some hints on when a state feedback yields Strong iISS, it does not provide any constructive way to design the corresponding control law. In this section, we rely on the so-called "universal" construction of Arstein's theorem to construct a bounded static state feedback ensuring Strong iISS. This construction relies on the knowledge of a control Lyapunov function (CLF), whose definition is recalled below (Lin and Sontag, 1991).

Definition 4 (CLF) A smooth proper storage function $V: \mathbb{R}^{n} \rightarrow \mathbb{R}_{\geq 0}$ is called a control Lyapunov function with controls in the unit ball for the (disturbance-free) system $\dot{x}=f(x)+g(x) u$ if it satisfies:

$$
\inf _{|u|<1}\left\{L_{f} V(x)+L_{g} V(x) u\right\}<0, \quad \forall x \neq 0
$$

In other words, a CLF with controls in the unit ball is a storage function whose total derivative can be point-wisely assigned to a negative value for each non-zero state by a control with amplitude lower than 1 . We may also require that this point-wise assignment be achievable by arbitrarily small control values, provided that the state is sufficiently close to the origin: this property is known as the small control property (SCP, Sontag (1989b)).

Definition 5 (SCP) A CLF $V: \mathbb{R}^{n} \rightarrow \mathbb{R}_{\geq 0}$ is said to satisfy the small control property for the (disturbance-free) system $\dot{x}=f(x)+g(x) u$ if, given any $\varepsilon>0$, there exists $\delta>0$ such that, if $|x|<\varepsilon$ and $x \neq 0$, then there exists $|u|<\delta$ such that $L_{f} V(x)+L_{g} V(x) u<0$.

We stress that, unlike other robust CLF proposed in the literature (Freeman and Kokotović, 1996; Teel and Praly, 2000; Liberzon et al., 2002; Liberzon, 2002), the above definitions are given for disturbance-free systems: the goal 
here is to provide a growth restriction on the function $h$ so that the bounded control law proposed in Lin and Sontag (1991) ensures Strong iISS for (6).

More precisely, the main contribution of the work Lin and Sontag (1991) is to propose an explicit continuous state feedback law, smooth out of the origin and of amplitude smaller than 1, that globally asymptotically stabilizes the disturbance-free system $\dot{x}=f(x)+g(x) u$. This state-feedback law reads $k(x)=\kappa\left(L_{f} V(x),\left|L_{g} V(x)\right|^{2}\right) L_{g} V(x)^{T}$, where $\kappa$ is defined, for each $(a, b) \in \mathbb{R} \times \mathbb{R}_{\geq 0}$, as

$$
\kappa(a, b):= \begin{cases}-\frac{a+\sqrt{a^{2}+b^{2}}}{b(1+\sqrt{1+b})} & \text { if } b>0 \\ 0 & \text { if } b=0\end{cases}
$$

The following result, proved in Section 5.2, states that this control law may be used as such to make the system (6) Strongly iISS provided a growth restriction on the function $h$.

Theorem 1 Let $V$ be a CLF with controls in the unit ball, satisfying the SCP for the disturbance-free system $\dot{x}=f(x)+g(x) u$. Assume that there exists $\alpha \in \mathcal{K}$ such that, for all $x \neq 0$,

$$
\liminf _{|x| \rightarrow \infty} \frac{\left|L_{f} V(x)\right|+\left|L_{g} V(x)\right|}{\left|L_{h} V(x)\right|}>0 .
$$

Assume also that

$$
\liminf _{\substack{|x| \rightarrow \infty \\ L_{f} V(x)>0}}\left|L_{g} V(x)\right|>0
$$

and

$$
\limsup _{|x| \rightarrow \infty} \frac{\left|L_{g} V(x)\right|}{\left|L_{f} V(x)\right|} \neq 1
$$

Then, under Assumption 2, the feedback law proposed in Lin and Sontag (1991), namely

$$
u=k(x)=\kappa\left(L_{f} V(x),\left|L_{g} V(x)\right|^{2}\right) L_{g} V(x)^{T},
$$

where $\kappa$ is defined in (11), is continuous on $\mathbb{R}^{n}$, smooth on $\mathbb{R}^{n} \backslash\{0\}$, has norm smaller than 1 and makes the saturated-actuation system (6) Strongly iISS.

Condition (12) and Assumption 2 express a growth rate limitation on the term $h(x)$ through which the perturbation acts on the system. Condition (13) is fairly intuitive: in the presence of a non-vanishing perturbation term and when the drift term is not dissipating, the control field needs to be non-vanishing as well in order to compensate these disturbances and ensure the state boundedness (hence Strong iISS). Condition (14) is far less intuitive; this technical assumption is required to prevent the dissipation rate to vanish when the state norm tends to infinity: see the proof for more details.

\subsection{ISS stabilization by "universal" constructions}

If $\alpha \in \mathcal{K}_{\infty}$ in the above statement, then it can be seen along the proof that the system (6) in closed loop with the same static feedback $k(x)$ results ISS. This observation complements the results in the literature that rely on the notion of ISS-CLF (Liberzon et al., 2002; Liberzon, 2002; Teel and Praly, 2000).

Corollary 1 If the assumptions of Theorem 1 hold with a $\mathcal{K}_{\infty}$ function $\alpha$, then the static state feedback law (15) makes the system (6) ISS. 


\section{4 iISS stabilization by "universal" constructions}

The assumptions of Theorem 1 can be considerably relaxed if only iISS is needed. We state this fact in the following corollary, whose proof is provided in Section 5.3.

Corollary 2 Let $V$ be a CLF with controls in the unit ball satisfying the SCP for the disturbance-free system $\dot{x}=f(x)+g(x) u$ and let Assumption 2 hold. Then the static state feedback law (15) makes the system (6) iISS.

Similarly to Theorem 1 , this result provides a growth rate limitation on the term $h(x)$ in such a way that the control law originally proposed in Lin and Sontag (1991) for disturbance-free systems, also yields a robustness property to exogenous disturbances. We stress, however, that the robustness property ensured by Corollary 2 (namely, iISS) is much weaker than that guaranteed by Theorem 1 (namely, Strong iISS) as it implies neither solutions' boundedness in response to sufficiently small disturbances nor state convergence in response to a vanishing perturbations.

\subsection{Example: spacecraft velocity control}

We now provide an illustration of the results obtained so far by considering the control of a rotating spacecraft through limited-thrust actuators. Letting $x:=\left(x_{1}, x_{2}, x_{3}\right)^{T}$ denote its the angular velocity and $u:=\left(u_{1}, u_{2}, u_{3}\right)^{T}$ the control torques, the dynamics is ruled by the following equations (Khalil, 2002):

$$
\begin{aligned}
& J_{1} \dot{x_{1}}=\left(J_{2}-J_{3}\right) x_{2} x_{3}+\bar{u} \operatorname{sat}^{0}\left(u_{1} / \bar{u}\right)+d_{1} \\
& J_{2} \dot{x_{2}}=\left(J_{3}-J_{1}\right) x_{3} x_{1}+\bar{u} \operatorname{sat}^{0}\left(u_{2} / \bar{u}\right)+d_{2} \\
& J_{3} \dot{x_{3}}=\left(J_{1}-J_{2}\right) x_{1} x_{2}+\bar{u} \operatorname{sat}^{0}\left(u_{3} / \bar{u}\right)+d_{3},
\end{aligned}
$$

where $d:=\left(d_{1}, d_{2}, d_{3}\right)^{T}$ represents exogenous perturbations (e.g. actuation errors) and $\bar{u}$ denotes the maximum thrust that can be delivered by each actuator. We use the Lyapunov function $V(x)=\frac{1}{2} x^{T} P x$, where $P:=\operatorname{diag}\left(J_{1}, J_{2}, J_{3}\right)$. Using the notation of (6), straightforward computations lead to $L_{f} V(x)=0, L_{g} V(x)=-\left(\bar{u} x_{1}, \bar{u} x_{2}, \bar{u} x_{3}\right)$ and $L_{h} V(x)=\left(x_{1}, x_{2}, x_{3}\right)$. It can easily be seen that $V$ is a CLF with controls in the unit ball for (16) and that it satisfies the SCP. It is also straightfoward to show that (12), (13) and (14) hold for this system. Theorem 1 can thus be invoked to design a bounded continuous feedback for Strong iISS, of the form $u=k_{\text {univ }}(x):=\kappa\left(0,\left|L_{g} V\right|^{2}\right) L_{g} V^{T}$, where $\kappa$ is given in (11). In other words:

$$
k_{u n i v}(x)=\frac{-\bar{u} x}{1+\sqrt{1+\bar{u}^{2}|x|^{2}}}
$$

The fact that $L_{f} V(x)=0$ allows in this case to make use of another control law, namely a saturated linear control:

$$
u=k_{l i n}(x):=\left(-k_{1} x_{1},-k_{2} x_{2},-k_{3} x_{3}\right)^{T},
$$

where $k_{1}, k_{2}, k_{3}$ denote positive gains. It can easily be shown that this linear control law makes the system (16) ISS in the absence of actuator saturation. We claim that, in the presence of limited thrust (namely $\left|u_{i}\right| \leq \bar{u}$ for each $i \in\{1,2,3\}$ ), the system results Strongly iISS with input threshold $R=\bar{u} / \sqrt{3}$. Indeed, in the presence of such saturating thrusters, the dynamics reads

$$
\begin{aligned}
& J_{1} \dot{x_{1}}=\left(J_{2}-J_{3}\right) x_{2} x_{3}-\bar{u} \operatorname{sat}^{0}\left(\tilde{k}_{1} x_{1}\right)+d_{1} \\
& J_{2} \dot{x_{2}}=\left(J_{3}-J_{1}\right) x_{3} x_{1}-\bar{u} \operatorname{sat}^{0}\left(\tilde{k}_{2} x_{2}\right)+d_{2} \\
& J_{3} \dot{x_{3}}=\left(J_{1}-J_{2}\right) x_{1} x_{2}-\bar{u} \operatorname{sat}^{0}\left(\tilde{k}_{3} x_{3}\right)+d_{3},
\end{aligned}
$$

where $\tilde{k}_{i}:=k_{i} / \bar{u}$ for each $i \in\{1,2,3\}$. Consequently, noticing that $\left|L_{h} V(x)\right| \neq 0$ for all $x \neq 0$, it holds that

$$
\begin{aligned}
& \frac{L_{f} V+L_{g} V \operatorname{sat}(k(x) / \bar{u})}{\left|L_{h} V\right|}= \\
& -\frac{x_{1} \operatorname{sat}\left(\tilde{k}_{1} x_{1}\right)+x_{2} \operatorname{sat}\left(\tilde{k}_{2} x_{2}\right)+x_{3} \operatorname{sat}\left(\tilde{k}_{3} x_{3}\right)}{|x|} \bar{u}
\end{aligned}
$$


which is clearly a negative definite function, thus establishing (7). Moreover, letting $|x|_{\infty}:=\max \left\{\left|x_{1}\right|,\left|x_{2}\right|,\left|x_{3}\right|\right\}$ and $\underline{k}:=\min \left\{\tilde{k}_{1}, \tilde{k}_{2}, \tilde{k}_{3}\right\}$, it holds that

$$
\begin{array}{r}
\frac{x_{1} \operatorname{sat}^{0}\left(\tilde{k}_{1} x_{1}\right)+x_{2} \operatorname{sat}^{0}\left(\tilde{k}_{2} x_{2}\right)+x_{3} \operatorname{sat}^{0}\left(\tilde{k}_{3} x_{3}\right)}{|x|} \\
\geq \frac{|x|_{\infty} \operatorname{sat}^{0}\left(\underline{k}|x|_{\infty}\right)}{|x|} .
\end{array}
$$

Observing that $|x| \leq|x|_{\infty} \sqrt{3}$, we obtain that

$$
\frac{L_{f} V+L_{g} V \operatorname{sat}\left(k_{l i n}(x) / \bar{u}\right)}{\left|L_{h} V\right|} \leq-\frac{\bar{u}}{\sqrt{3}} \operatorname{sat}^{0}(\underline{k}|x| / \sqrt{3}),
$$

which makes (8) fulfilled with $\gamma(s)=\operatorname{sat}^{0}(\underline{k} s / \sqrt{3}) \bar{u} / \sqrt{3}$. Thus, Assumption 1 is satisfied. Assumption 2 being trivially satisfied in this case (since $\left|L_{h} V\right|=|x|$ ), we conclude from Lemma 1 that, as claimed, the system is Strongly iISS with input threshold $\gamma(\infty)=\bar{u} / \sqrt{3}$.

We tested numerically the performance of both the feedback law $k_{u n i v}(x)$ proposed in $(17)$ and the saturated linear control law $k_{\text {lin }}(x)$ provided in (18), for the following parameter values: $J_{1}=J_{2}=5 \mathrm{~kg} . \mathrm{m}^{2}, J_{3}=10 \mathrm{~kg} . \mathrm{m}^{2}$, $\bar{u}=1 \mathrm{~kg} \cdot \mathrm{m}^{3} \cdot \mathrm{s}^{-2}$, and $k_{1}=k_{2}=k_{3}=5 \mathrm{~kg} \cdot \mathrm{m}^{2} \cdot \mathrm{s}^{-1}$. As expected, as long as the disturbance norm $|d|$ is below $1 / \sqrt{3} \mathrm{~kg} \cdot \mathrm{m}^{3} \cdot \mathrm{s}^{-2}$ the satellite angular velocities reaches the desired configuration $x_{1}=x_{2}=x_{3}=0 \mathrm{~m} \cdot \mathrm{s}^{-1}$ up to a steady-state error proportional to the disturbance amplitude, with both control laws: see Figure 1. Both the control amplitudes remain below $\bar{u}$. A better performance is achieved by the saturated linear controller, but this is at the price of more solicitation of the thrusters.
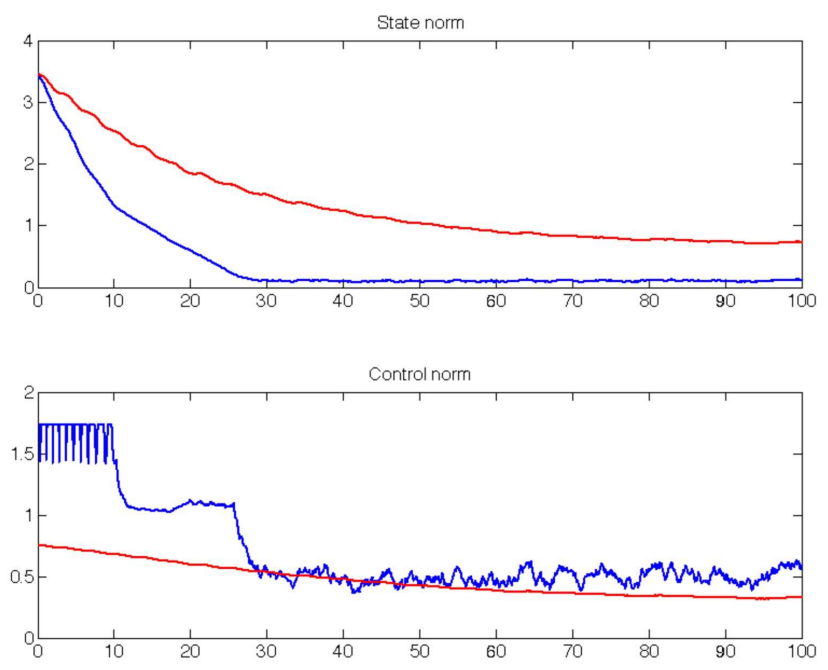

Fig. 1. State and control magnitude of the spacecraft for a random disturbance with magnitude below $1 / \sqrt{3} \mathrm{~kg} \cdot \mathrm{m}^{3} \cdot \mathrm{s}^{-2}$. Blue: saturated linear feedback $k_{\text {lin }}(x)$ in (18), red: universal law $k_{u n i v}(x)$ in $(17)$.

Simulations also show that the input threshold of $1 / \sqrt{3}$ is a reasonable estimate of the actual disturbance amplitude that can be tolerated by the spacecraft. For instance, when considering zero disturbances on the $x_{1}$ and $x_{2}$ axes $\left(d_{1}=d_{2}=0\right)$, the systems keeps on having a bounded steady-state response for a constant disturbance $d_{3}$ below $1 / \sqrt{3} \mathrm{~kg} \cdot \mathrm{m}^{3} \cdot \mathrm{s}^{-2}$ with both the control laws, while the universal control law (17) yields unbounded response above that value. However, for the saturated linear state feedback (18), the system seems to stand disturbances up to $d_{3}=1 \mathrm{~kg} \cdot \mathrm{m}^{3} \cdot \mathrm{s}^{-2}$. The corresponding plots are omitted due to space constraints.

\section{LTI systems: the neutrally stable case}

As illustrated by the spacecraft example of Section 3.5, saturated linear feedback may provide better performance than the "universal" constructions studied in the previous section. Among the systems that can be stabilized by 
saturated linear state feedback, we next focus on the class of neutrally stable systems affected by actuator saturation and exogenous disturbances:

$$
\dot{x}=A x-B \sigma(u)+d .
$$

We recall that a neutrally stable matrix is any matrix $A$ such that the solutions of $\dot{x}=A x$ are bounded. This is equivalent to requiring that there exists a symmetric positive definite matrix $P$ such that $A^{T} P+P A$ is a positive semi-definite matrix. For the sake of generality, the function $\sigma: \mathbb{R}^{m} \rightarrow \mathbb{R}^{m}$ is assumed to belong to the class $\mathcal{S}^{m}$, as defined below (Liu et al., 1996).

Definition 6 ( $\mathcal{S}$ and $\mathcal{S}^{m}$ functions) $\mathcal{S}$ denotes the set of all locally Lipschitz bounded function $S: \mathbb{R} \rightarrow \mathbb{R}$ satisfying: $S(0)=0, S(s) s>0$ for all $s \neq 0, \liminf _{s \rightarrow 0} S(s) / s>0$, and $\liminf _{|s| \rightarrow \infty}|S(s)|>0$. A vector function $\sigma: \mathbb{R}^{m} \rightarrow \mathbb{R}^{m}$ is said to be in the class $\mathcal{S}^{m}$ if it reads $\sigma(u)=\left(S_{1}\left(u_{1}\right), \ldots, S_{m}\left(u_{m}\right)\right)^{T}$, for all $u \in \mathbb{R}^{m}$, where $S_{i} \in \mathcal{S}$ for each $i \in\{1, \ldots, m\}$.

We stress that the class $\mathcal{S}^{m}$ includes, but is not limited to, sat functions. For more details about the functions included in this class, the reader is invited to refer to Liu et al. (1996). We state the following useful property of $\mathcal{S}^{m}$-functions. It is a straightforward $m$-dimensional extension of (Liu et al., 1996, Remark 2).

Fact 1 If $\sigma \in \mathcal{S}^{m}$, then there exist constants $a, b, K>0$ and a measurable diagonal-matrix valued function $\tau: \mathbb{R}^{m} \rightarrow$ $\mathbb{R}^{m \times m}$ such that, for all $u \in \mathbb{R}^{m}$,

$$
a I \leq \tau(u) \leq b I, \quad|\sigma(u)-\tau(u) u| \leq K u \sigma(u) .
$$

\subsection{From neutral stability to skew-symmetry}

Reasoning as in (Liu et al., 1996, Section 3.2) the question of robustly stabilizing (20) when $A$ is neutrally stable boils down to the case when $A$ is skew-symmetric. This comes from the fact that any neutrally stable matrix is similar to $\operatorname{diag}\left(A_{H}, A_{S}\right)$ where $A_{H}$ is a Hurwitz matrix and $A_{S}$ is skew-symmetric. This lets us consider that $A$ is skew-symmetric without loss of generality.

\subsection{Strong iISS by saturated linear feedback}

Beyond $L_{p}$-stability results for disturbances entering the function $\sigma$, it was shown in Liu et al. (1996) that the linear state feedback $u=B^{T} x$ globally asymptotically stabilizes the saturated system (20) in the absence of exogenous disturbances. Theorem 2 in Liu et al. (1996) also shows that solutions are bounded if the disturbance $d$ is of sufficiently low amplitude. The following result unifies and goes further these observations, by establishing that the same feedback law ensures Strong iISS to the system (20).

Theorem 2 Let $\sigma \in \mathcal{S}^{m}$ and assume that $A \in \mathbb{R}^{n \times n}$ is skew-symmetric and that the pair $(A, B)$ is controllable. Then the linear static state feedback $u=B^{T} x$ makes the saturated system (20) Strongly iISS.

The proof of this result, provided in Section 5.4, relies on the following technical result.

Lemma 2 Let $A \in \mathbb{R}^{n \times n}$ be a skew-symmetric matrix and $B \in \mathbb{R}^{n \times m}$ be such that the pair $(A, B)$ is controllable. Let $D: \mathbb{R}_{\geq 0} \rightarrow \mathbb{R}^{m \times m}$ be any bounded measurable matrix-valued function satisfying $D(t)+D(t)^{T} \geq \varepsilon I$ for almost all $t \in \mathbb{R}_{\geq 0}$, where $\varepsilon$ denotes a positive constant. Then, there exists a symmetric positive definite matrix $P \in \mathbb{R}^{n \times n}$ such that, for almost all $t \in \mathbb{R}_{\geq 0}$,

$$
\left(A-B D(t) B^{T}\right)^{T} P+P\left(A-B D(t) B^{T}\right) \leq-I .
$$

Moreover, this matrix $P$ can be picked as $P=P_{0}+\chi I$, where $P_{0} \in \mathbb{R}^{n \times n}$ denotes any symmetric positive definite matrix satisfying

$$
\left(A-\varepsilon B B^{T}\right)^{T} P_{0}+P_{0}\left(A-\varepsilon B B^{T}\right) \leq-2 I,
$$


as ensured by the controllability of $(A, B)$, and $\chi$ denotes any constant satisfying

$$
\chi \geq \frac{1}{\varepsilon} \sup _{t \geq 0}\left|P_{0} B(\varepsilon I-D(t))\right|
$$

The proof of Lemma 2 can be found along the lines of (Liu et al., 1996, Lemma 3.2 and Corollary 1) and is therefore omitted.

\subsection{Estimate of the input threshold}

The proof of Theorem 2 is constructive: once a matrix $P$ such as the one generated by Lemma 2 is known, we can estimate the resulting input threshold for (20). These findings are summarized by the following result.

Corollary 3 Let $\sigma \in \mathcal{S}^{m}$ and let $A \in \mathbb{R}^{n \times n}$ be a skew-symmetric matrix such that the pair $(A, B)$ is controllable. Let $a, b$ and $K$ be any positive constants such that (21) holds for all $u \in \mathbb{R}^{m}$, where $\tau: \mathbb{R}^{m} \rightarrow \mathbb{R}^{m \times m}$ is the measurable diagonal-matrix valued function. Let $P_{0} \in \mathbb{R}^{n \times n}$ be any symmetric positive definite matrix such that

$$
\left(A-2 a B B^{T}\right)^{T} P_{0}+P_{0}\left(A-2 a B B^{T}\right) \leq-2 I .
$$

Finally, let $\chi$ be the positive constant defined as

$$
\chi=\frac{|2 a-b|}{2 a}\left|P_{0} B\right|
$$

and let $P:=P_{0}+\chi I$. Then (20) in closed loop with the control law $u=B^{T} x$ is Strongly iISS with input threshold

$$
R=\frac{1}{2 K|P B|+|P|(4 K|P B| / 3)^{1 / 3}}
$$

Note that the existence of such constants $a, b, k$ and such a function $\tau$ is ensured by Fact 1 . The existence of a matrix $P_{0}$ satisfying $(24)$ is guaranteed by the controllability of the pair $(A, B)$. The proof of this result follows straightforwardly from that of Theorem 2 . We provide its main steps in Section 5.5 for the sake of completeness.

For some particular saturations, the function $\tau$ of Fact 1 can be picked as a constant matrix. This includes for instance saturation functions of the form:

$$
S_{i}(s)=\operatorname{sat}_{\ell}(s):=\operatorname{sign}(s) \min \{\ell|s| ; 1\}, \quad \forall s \in \mathbb{R},
$$

where $\ell$ denotes its linear slope (note that, when $\ell=1$, we recover the sat ${ }^{0}$ function used all along Section 3 ). For such functions, the expression of the input threshold estimate is slightly simpler. We summarize this in the following corollary.

Corollary 4 Let $S \in \mathcal{S}^{m}$ and let $A \in \mathbb{R}^{n \times n}$ be a skew-symmetric matrix such that the pair $(A, B)$ is controllable. Assume that there exist $\bar{\tau}, K>0$ such that

$$
|\sigma(u)-\bar{\tau} u| \leq K u \sigma(u), \quad \forall u \in \mathbb{R}^{m}
$$

Let $P_{0} \in \mathbb{R}^{n \times n}$ be any symmetric positive definite matrix such that

$$
\left(A-2 \bar{\tau} B B^{T}\right)^{T} P_{0}+P_{0}\left(A-2 \bar{\tau} B B^{T}\right) \leq-2 I,
$$

as ensured by the controllability of the pair $(A, B)$. Finally, let $P:=P_{0}+\frac{1}{2}\left|P_{0} B\right| I$. Then the system (20) in closed loop with the control law $u=B^{T} x$ is Strongly iISS with the input threshold given in (26). 


\subsection{Example: the harmonic oscillator}

We finally illustrate the above results with the following planar system:

$$
\left(\begin{array}{c}
\dot{x}_{1} \\
\dot{x}_{2}
\end{array}\right)=\left(\begin{array}{cc}
0 & 1 \\
-1 & 0
\end{array}\right)\left(\begin{array}{l}
x_{1} \\
x_{2}
\end{array}\right)-\left(\begin{array}{l}
0 \\
1
\end{array}\right) \operatorname{sat}_{\ell}(u)+d,
$$

where $\ell>0$. This system is in the form of (20) with

$$
A=\left(\begin{array}{cc}
0 & 1 \\
-1 & 0
\end{array}\right), \quad B=\left(\begin{array}{l}
0 \\
1
\end{array}\right), \quad \sigma(s)=\operatorname{sat}_{\ell}(s) .
$$

Note that the perturbation is not matching, as it affects the dynamics of $x_{1}$ although no control is available in that direction. The pair $(A, B)$ being controllable, we conclude with Theorem 2 that the state feedback $u=B^{T} x=x_{2}$ makes the system (30) Strong iISS. In addition, we can estimate its input threshold by invoking Corollary 4 . To that end, notice that $\left|\operatorname{sat}_{\ell}(s)-\ell s\right| \leq \ell s \operatorname{sat}_{\ell}(s)$ for all $s \in \mathbb{R}$, meaning that (28) is satisfied with $\bar{\tau}=K=\ell$. Furthermore, the Lyapunov equation (29) is satisfied with the following symmetric positive definite matrix:

$$
P_{0}=\frac{1}{2}\left(\begin{array}{cc}
2 \ell+1 / \ell & 1 \\
1 & 1 / \ell
\end{array}\right) .
$$

Noticing that $\left|P_{0} B\right|=\frac{1}{2} \sqrt{1+\ell^{2}}$, the input threshold of (30) in closed loop with $u=B^{T} x=x_{2}$ can be computed as a function of the slope $\ell$ of the saturation according to the estimate (26). We obtain the curve reported in Figure 2.

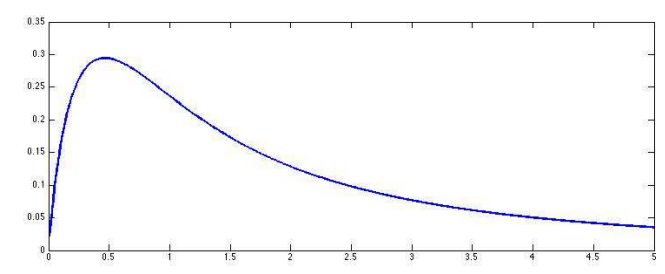

Fig. 2. Input threshold estimate of the planar system (30) as a function of the slope $\ell$ of the saturation.

This plot shows that the estimate of $R$ provided by Corollary 4 is not accurate for this specific example, as it tends to zero for large values of the slope $\ell$ (meaning when sat $\ell$ tends to the sign function). Simulations suggest that the actual input threshold rather tends to 1. Although providing an explicit estimate of the input threshold, the tools employed in this paper fail at providing a tighter bound. The authors believe that a better choice of the feedback law than merely $u=B^{T} x$ would allow a better estimate of the guaranteed input threshold, but this goes beyond the scope of the paper.

\section{Proofs}

\subsection{Proof of Lemma 1}

The total derivative of $V$ along the solutions of (6) in closed loop with $u=k(x)$ reads

$$
\begin{aligned}
\dot{V} & =L_{f} V(x)+L_{g} V(x) k(x)+L_{h} V(x) d \\
& \leq L_{f} V(x)+L_{g} V(x) k(x)+\left|L_{h} V(x)\right||d| .
\end{aligned}
$$

In view of (8) in Assumption 1 it holds that, for all $x \neq 0$,

$$
\left.\begin{array}{c}
L_{h} V(x) \neq 0 \\
|d| \leq \gamma(|x|)
\end{array}\right\} \Rightarrow \dot{V}<0 .
$$


Moreover, from (7), it holds that, for all $x \neq 0$,

$$
L_{h} V(x)=0 \quad \Rightarrow \quad \dot{V}<0 .
$$

Therefore, noticing that $\dot{V}=0$ for $x=0$, there exists a $\mathcal{P D}$ function $\rho$ such that, for all $x \in \mathbb{R}^{n}$ and all $d \in \mathbb{R}^{p}$,

$$
|d| \leq \gamma(|x|) \quad \Rightarrow \quad \dot{V} \leq-\rho(|x|)
$$

We now rely on the following result.

Proposition 1 Assume that there exist a proper storage function $V: \mathbb{R}^{n} \rightarrow \mathbb{R}_{\geq 0}$, a class $\mathcal{K}$ function $\gamma$ and a $\mathcal{P D}$ function $\rho$ such that, for all $x \in \mathbb{R}^{n}$ and all $d \in \mathbb{R}^{p}$,

$$
|d| \leq \gamma(|x|) \quad \Rightarrow \quad \frac{\partial V}{\partial x}(x) f(x, d) \leq-\rho(|x|) .
$$

Then (20) is ISS with respect to inputs $d \in \mathcal{U}_{<\gamma(\infty)}^{p}$.

The proof of this proposition follows from typical manipulations on ISS Lyapunov functions and is therefore omitted. We stress that, in the case when $\gamma \in \mathcal{K}_{\infty}$, we recover a classical ISS characterization (Sontag and Wang, 1995).

Invoking Proposition 1 we conclude from (31) that, under the control law $u=k(x)$, the system (6) is ISS with respect to all $d \in \mathcal{U}_{<\gamma(\infty)}^{p}$. Consequently, there is only left to prove that this system is iISS. To this aim, let $W(x):=\ln (1+V(x))$. Noticing that Assumption 1 ensures the existence of a $\mathcal{P D}$ function $\tilde{\rho}$ such that $L_{f} V(x)+$ $L_{g} V(x) k(x) \leq-\tilde{\rho}(|x|)$, we get that

$$
\begin{aligned}
\dot{W} & =\frac{L_{f} V(x)+L_{g} V(x) k(x)+L_{h} V(x) d}{1+V(x)} \\
& \leq-\frac{\tilde{\rho}(|x|)}{1+V(x)}+\frac{\left|L_{h} V(x)\right|}{1+V(x)}|d| .
\end{aligned}
$$

In view of (10), which is ensured by Assumption 2, we obtain that

$$
\dot{W} \leq-\frac{\tilde{\rho}(|x|)}{1+V(x)}+K|d| .
$$

Noting that $x \mapsto \frac{\tilde{\rho}(|x|)}{1+V(x)}$ is a $\mathcal{P} \mathcal{D}$ function we can thus deduce by Angeli et al. (2000) that the system is iISS. We conclude that the system is indeed Strongly iISS with input threshold $\gamma(\infty)$.

\subsection{Proof of Theorem 1}

For notation simplicity, let $a(x):=L_{f} V(x)$ and $b(x):=\left|L_{g} V(x)\right|^{2}$. The smoothness and magnitude properties of the feedback law $k(x)$ are established in Lin and Sontag (1991). Moreover, since $|k(x)| \leq 1$, it holds that

$$
\begin{aligned}
L_{f} V+L_{g} V \operatorname{sat}(k(x)) & =L_{f} V+L_{g} V k(x) \\
& =a(x)+b(x) \kappa(a(x), b(x)) .
\end{aligned}
$$

In view of Lemma 1, since Assumption 2 holds, only Assumption 1 needs to be checked. Since the feedback law proposed in Lin and Sontag (1991) ensures $a(x)+b(x) \kappa(a(x), b(x))<0$ for all $x \neq 0$, all we need to show is (8), that is

$$
|a(x)+b(x) \kappa(a(x), b(x))|>\tilde{\alpha}(|x|)\left|L_{h} V(x)\right|,
$$

for some $\mathcal{K}$ function $\tilde{\alpha}$. To that end, first notice that

$$
|a(x)|+b(x) \neq 0, \quad \forall x \neq 0
$$


indeed, if $a(x) \neq 0$ this holds trivially, and if $a(x)=0$ it holds that $b(x) \neq 0$ since $V$ is a CLF. Recalling that $b(x) \geq 0$, this in turn implies that

$$
|a(x)|+\frac{b(x)}{\sqrt{1+b(x)}} \neq 0, \quad \forall x \neq 0 .
$$

Moreover, (12) ensures that

$$
\liminf _{|x| \rightarrow \infty} \frac{1}{\left|L_{h} V(x)\right|}\left(|a(x)|+\frac{b(x)}{\sqrt{1+b(x)}}\right)>0 .
$$

To see this, notice that (12) implies that either $\liminf _{|x| \rightarrow \infty} \frac{|a(x)|}{\left|L_{h} V(x)\right|}>0$ or $\liminf _{|x| \rightarrow \infty} \frac{|b(x)|}{\left|L_{h} V(x)\right|}>0$. In the first case, (34) trivially holds. In the second case, it holds that $\liminf _{|x| \rightarrow \infty}|b(x)| /\left|L_{h} V(x)\right| \sqrt{1+b(x)}>0$, which also makes (34) fulfilled. Combining (33) and (34) ensures the existence of a class $\mathcal{K}$ function $\alpha$ such that

$$
\left|L_{h} V(x)\right| \alpha(|x|)<|a(x)|+\frac{b(x)}{\sqrt{1+b(x)}}, \quad \forall x \neq 0 .
$$

Furthermore, omitting the $x$-dependency in the notation and recalling that $a+b \kappa(a, b)<0$, it holds that

$$
\begin{aligned}
|a+b \kappa(a, b)| & =\left|\frac{a \sqrt{1+b}-\sqrt{a^{2}+b^{2}}}{1+\sqrt{1+b}}\right| \\
& =\frac{\sqrt{1+b}}{1+\sqrt{1+b}}\left|a-\sqrt{\frac{a^{2}+b^{2}}{1+b}}\right| \\
& >\frac{1}{2}\left|a-\sqrt{\frac{a^{2}+b^{2}}{1+b}}\right|
\end{aligned}
$$

where the last bound comes from the fact that $b \mapsto \sqrt{1+b} /(1+\sqrt{1+b})$ is greater than $1 / 2$ over $\mathbb{R}_{>0}$. Now, pick any $x \neq 0$ and consider the following cases.

Case 1: $b(x)=0$. Then (35) implies that $|a(x)|>\alpha(|x|)\left|L_{h} V(x)\right|$. Consequently

$$
|a(x)+b(x) \kappa(a(x), b(x))|=|a(x)|>\alpha(|x|)\left|L_{h} V(x)\right|
$$

Case $2: b(x) \neq 0, a(x) \leq 0$. Then we have from (36) that

$$
\begin{aligned}
|a+b \kappa(a, b)| & >\frac{1}{2}\left(\sqrt{\frac{a^{2}+b^{2}}{1+b}}+|a|\right) \\
& >\frac{1}{2}\left(\frac{b}{\sqrt{1+b}}+|a|\right) .
\end{aligned}
$$

We thus obtain from (35) that

$$
|a+b \kappa(a, b)|>\frac{\alpha(|x|)}{2}\left|L_{h} V(x)\right|
$$

Case 3: $b(x) \neq 0$ and $a(x)>0$. The fact that $V$ is a CLF with $u$ constrained in the unit ball guarantees that

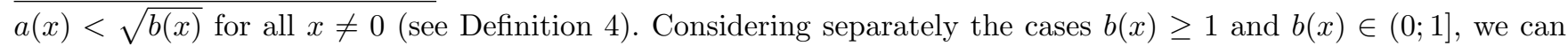
easily deduce that the following bound holds:

$$
\frac{3 \max (b ; \sqrt{b})}{\sqrt{1+b}}>a+\frac{b}{\sqrt{1+b}}
$$


It follows from (35) that

$$
\frac{3 \max (b ; \sqrt{b})}{\sqrt{1+b}}>\alpha(|x|)\left|L_{h} V(x)\right|
$$

Moreover, from (36), we have that

$$
\begin{aligned}
|a+b \kappa(a, b)| & >\frac{1}{2}\left(\sqrt{\frac{a^{2}+b^{2}}{1+b}}-a\right) \\
& =\frac{1}{2} \sqrt{\frac{a^{2}+b^{2}}{1+b}}\left(1-\sqrt{\frac{a^{2}+a^{2} b}{a^{2}+b^{2}}}\right) \\
& >\frac{b}{2 \sqrt{1+b}}\left(1-\sqrt{\frac{1+b}{1+b^{2} / a^{2}}}\right) .
\end{aligned}
$$

First consider the case when $b(x) \geq 1$. Then $(39)$ reads $3 b / \sqrt{1+b}>\alpha(|x|)\left|L_{h} V(x)\right|$ and we get that

$$
\frac{b}{2 \sqrt{1+b}}>\frac{\alpha(|x|)\left|L_{h} V(x)\right|}{6} \text {. }
$$

On the other hand, if $b(x) \in(0 ; 1]$, then $(39) \operatorname{reads} 3 \sqrt{b} / \sqrt{1+b}>\alpha(|x|)\left|L_{h} V(x)\right|$ and we get from (40) that

$$
\frac{b}{2 \sqrt{1+b}}>\frac{\alpha(|x|)\left|L_{h} V(x)\right|}{6} \sqrt{b}
$$

The assumption (13) implying the existence of a class $\mathcal{K}$ function $\mu$ such that $\sqrt{b}=\left|L_{g} V(x)\right| \geq \mu(|x|)$, we obtain from (40) and (41)-(42) that

$$
|a+b \kappa(a, b)|>\bar{\alpha}(|x|)\left|L_{h} V\right|\left(1-\sqrt{\frac{1+b}{1+b^{2} / a^{2}}}\right),
$$

where $\bar{\alpha}(\cdot):=\alpha(\cdot) \min \{\mu(\cdot) ; 1\} / 6$ is a class $\mathcal{K}$ function. We claim that there exists $\alpha^{\prime} \in \mathcal{K}$ such that

$$
1-\sqrt{\frac{1+b(x)}{1+b(x)^{2} / a(x)^{2}}} \geq \alpha^{\prime}(|x|)
$$

To see this first notice that, since $a(x)<\sqrt{b(x)}$, the above function is positive on $\mathbb{R}^{n} \backslash\{0\}$. Moreover, condition $(14)$ together with the fact that $0<a(x)<\sqrt{b(x)}$ ensures that $\limsup _{|x| \rightarrow \infty} b(x) / a(x)^{2}>1$. Consequently:

$$
\liminf _{|x| \rightarrow \infty}\left(1-\sqrt{\frac{1+b(x)}{1+b(x)^{2} / a(x)^{2}}}\right)>0 .
$$

We conclude that there indeed exists a function $\alpha^{\prime} \in \mathcal{K}$ satisfying (44). We get from (44) and (43) that

$$
|a(x)+b(x) \kappa(a(x), b(x))|>\alpha^{\prime \prime}(|x|)\left|L_{h} V(x)\right|
$$

where $\alpha^{\prime \prime}(\cdot):=\bar{\alpha}(\cdot) \alpha^{\prime}(\cdot)$ is also class $\mathcal{K}$.

Combining (37), (38) and (45), we conclude that (32) holds. The conclusion then follows from the application of Lemma 1. 


\subsection{Proof of Corollary 2}

Since $V$ is a CLF with controls in the unit ball satisfying the SCP for the disturbance-free system $\dot{x}=f(x)+g(x) u$, it was shown in Lin and Sontag (1991) that the control law (15) satisfies $L_{f} V(x)+L_{g} V(x) k(x)<0$ for all $x \neq 0$. Equivalently, there exists a continuous positive definite function $\rho \in \mathcal{P} \mathcal{D}$ such that $L_{f} V(x)+L_{g} V(x) k(x) \leq-\rho(|x|)$ for all $x \in \mathbb{R}^{n}$. Hence, the derivative of $W:=\ln (1+V)$ along the solutions of (6) satisfies

$$
\begin{aligned}
\dot{W} & =\frac{\dot{V}}{1+V(x)} \\
& =\frac{1}{1+V(x)}\left(L_{f} V(x)+L_{g} V(x) k(x)+L_{h} V(x) d\right) \\
& \leq-\frac{\rho(|x|)}{1+V(x)}+\frac{\left|L_{h} V(x)\right|}{1+V(x)}|d| .
\end{aligned}
$$

As already stressed, Assumption 2 implies the existence of a constant $K>0$ such that (10) holds. Consequently:

$$
\dot{W} \leq-\frac{\rho(|x|)}{1+V(x)}+K|d|
$$

The conclusion then follows by the classical Lyapunov characterization of iISS Angeli et al. (2000) after noticing that $x \mapsto \rho(|x|) /(1+V(x))$ is a $\mathcal{P} \mathcal{D}$ function.

\subsection{Proof of Theorem 2}

In order to establish Theorem 2, we make use of the Lyapunov function proposed in Liu et al. (1996). More precisely, letting

$$
V(x):=\frac{c}{3}|x|^{3}+x^{T} P x, \quad \forall x \in \mathbb{R}^{n},
$$

where $P \in \mathbb{R}^{n \times n}$ is a convenient symmetric positive definite matrix, and $c>0$ denotes some well chosen constant, we claim that the proper $C^{1}$ Lyapunov function candidate

$$
\tilde{V}(x):=\frac{1}{3}\left[(1+V(x))^{1 / 3}-1\right]
$$

satisfies, along the solutions of (20) in closed loop with $u=B^{T} x$,

$$
\dot{\tilde{V}}(x) \leq-W(x)+\gamma(|d|)
$$

where $\gamma$ denotes a class $\mathcal{K}_{\infty}$ function and $W$ is a continuous positive definite function satisfying $\lim \inf _{x \rightarrow \infty} W(x)>0$. According to (Chaillet et al., 2014a, Theorem 1), this establishes Strong iISS, as claimed in the statement.

To that aim, we start by decomposing the function $V$ as $V(x)=c V_{1}(x)+V_{2}(x)$, where $V_{1}(x):=|x|^{3} / 3$ and $V_{2}(x):=x^{T} P x$. We will study the time derivative of $V_{1}$ and $V_{2}$ along the solutions of (20) separately. We start by the function $V_{1}$ : recalling that $x^{T} A x=0$ for all $x \in \mathbb{R}^{n}$ (as $A$ is skew-symmetric), direct computations show that

$$
\begin{aligned}
\dot{V}_{1} & =|x| x^{T} \dot{x} \\
& =-|x| x^{T} B \sigma\left(B^{T} x\right)+|x| x^{T} d .
\end{aligned}
$$

In order to study the derivative of $V_{2}$, consider any initial state $x_{0} \in \mathbb{R}^{n}$ and any input signal $d \in \mathcal{U}^{n}$. Let $\tau$ be the function generated by Fact 1 and let

$$
\tilde{A}(t):=A-B \tau\left(B^{T} x(t)\right) B^{T}, \quad \forall t \geq 0
$$


where $x(\cdot)$ denotes the solution of (20) starting from $x_{0}$. Note that the saturation function $\sigma$ being locally Lipschitz and bounded, the existence of the solutions of (20) is ensured at all forward time, which makes the above matrix $\tilde{A}(t)$ well defined at all times $t \geq 0$. With this notation, the system (20) in closed loop with $u=B^{T} x$ can be rewritten as

$$
\dot{x}=\tilde{A}(t) x+B\left[\tau\left(B^{T} x\right) B^{T} x-\sigma\left(B^{T} x\right)\right]+d .
$$

Now, in view of Fact 1 and recalling that $\tau(u)$ is a diagonal matrix for each $u \in \mathbb{R}^{m}$, it holds that

$$
\tau\left(B^{T} x(t)\right)+\tau\left(B^{T} x(t)\right)^{T} \geq 2 a I, \quad \forall t \geq 0,
$$

for some constant $a>0$ independent of $x_{0}$. It follows that all the conditions of Lemma 2 are fulfilled with $D(t)=$ $\tau\left(B^{T} x(t)\right)$ and $\varepsilon=2 a$. Consequently, there exists a symmetric positive definite matrix $P=P^{T}$ such that

$$
\tilde{A}(t)^{T} P+P \tilde{A}(t) \leq-I, \quad \forall t \geq 0 .
$$

The derivative of the function $V_{2}(x)=x^{T} P x$ with this particular matrix $P$ along the solutions of (49) reads

$$
\begin{aligned}
\dot{V}_{2}= & x^{T}\left(\tilde{A}(t)^{T} P+P \tilde{A}(t)\right) x \\
& +2 x^{T} P B\left[\tau\left(B^{T} x\right) B^{T} x-\sigma\left(B^{T} x\right)\right]+2 x^{T} P d \\
\leq & -|x|^{2}+2|x||P B|\left|\tau\left(B^{T} x\right) B^{T} x-\sigma\left(B^{T} x\right)\right|+2 x^{T} P d .
\end{aligned}
$$

Recalling that, in view of Fact $1,\left|\tau\left(B^{T} x\right) B^{T} x-\sigma\left(B^{T} x\right)\right| \leq K x^{T} B \sigma\left(B^{T} x\right)$, it follows that

$$
\dot{V}_{2} \leq-|x|^{2}+2 K|x||P B| x^{T} B \sigma\left(B^{T} x\right)+2 x^{T} P d .
$$

Combining (48) and (50) and picking $c=2 K|P B|$, we obtain that the derivative of $V(x)=c V_{1}(x)+V_{2}(x)$ along the solutions of (20) satisfies

$$
\dot{V} \leq-|x|^{2}+c|x|^{2}|d|+2|P||x||d| .
$$

Thus the function $\tilde{V}$, defined in (46), satisfies

$$
\begin{aligned}
\dot{\tilde{V}}= & \frac{\dot{V}(x)}{[1+V(x)]^{2 / 3}} \\
\leq & -\frac{|x|^{2}}{[1+V(x)]^{2 / 3}}+c \frac{|x|^{2}}{[1+V(x)]^{2 / 3}}|d| \\
& +2|P| \frac{|x|}{[1+V(x)]^{2 / 3}}|d| .
\end{aligned}
$$

We now analyze the two nonnegative term of this upper bound separately. First, it holds that

$$
\begin{aligned}
\frac{|x|^{2}}{[1+V(x)]^{2 / 3}} & =\frac{|x|^{2}}{\left[1+x^{T} P x+\frac{c}{3}|x|^{3}\right]^{2 / 3}} \\
& \leq \frac{|x|^{2}}{\left[1+\frac{c}{3}|x|^{3}\right]^{2 / 3}} \\
& \leq\left(\frac{|x|^{3}}{1+\frac{c}{3}|x|^{3}}\right)^{2 / 3} \\
& \leq\left(\frac{3}{c}\right)^{2 / 3} .
\end{aligned}
$$


In the same way, we have that

$$
\begin{aligned}
\frac{|x|}{[1+V(x)]^{2 / 3}} & =\frac{|x|}{\left[1+x^{T} P x+\frac{c}{3}|x|^{3}\right]^{2 / 3}} \\
& \leq \frac{|x|}{\left[1+\frac{c}{3}|x|^{3}\right]^{2 / 3}} \\
& \leq\left(\frac{|x|^{3 / 2}}{1+\frac{c}{3}|x|^{3}}\right)^{2 / 3} \\
& \leq\left(\frac{3}{4 c}\right)^{1 / 3},
\end{aligned}
$$

where the last inequality follows form the fact that the function $s \mapsto s /\left(1+c s^{2} / 3\right)$ reaches its maximum $\sqrt{3 / 4 c}$ at $s=\sqrt{3 / c}$. Plugging (52)-(53) into (51) leads, as claimed, to

$$
\dot{\tilde{V}}(x) \leq-W(x)+\gamma(|d|)
$$

where $\gamma$ denotes the following $\mathcal{K}_{\infty}$ function:

$$
\gamma(s):=\left[(9 c)^{1 / 3}+|P|\left(\frac{6}{c}\right)^{1 / 3}\right] s, \quad \forall s \geq 0 .
$$

and $W: \mathbb{R}^{n} \rightarrow \mathbb{R}_{\geq 0}$ is the function defined as

$$
W(x):=\frac{|x|^{2}}{\left[1+x^{T} P x+\frac{c}{3}|x|^{3}\right]^{2 / 3}}, \quad \forall x \in \mathbb{R}^{n}
$$

where we recall that $c=2 K|P B|$. Notice that $W$ is continuous and positive definite and satisfies

$$
\liminf _{|x| \rightarrow \infty} W(x)=\liminf _{|x| \rightarrow \infty} \frac{|x|^{2}}{(c / 3)^{2 / 3}|x|^{2}}=\left(\frac{3}{c}\right)^{2 / 3} .
$$

Thus, we can apply (Chaillet et al., 2014a, Theorem 1) to conclude that (20) is Strongly iISS with input threshold

$$
\begin{aligned}
\gamma^{-1}\left((3 / c)^{2 / 3}\right) & =\frac{1}{c+|P|(2 c / 3)^{1 / 3}} \\
& =\frac{1}{2 K|P B|+|P|(4 K|P B| / 3)^{1 / 3}} .
\end{aligned}
$$

\subsection{Proof of Corollary 3}

Since $a I \leq \tau(u) \leq b I$, the constant $\chi$ introduced in (25) satisfies condition (23). By Lemma 2 , the proof of Theorem 2 can be repeated with $P=P_{0}+\chi I$. The rest of the proof is identical.

\section{Conclusion}

After having presented a sufficient condition for Strong iISS under saturated feedback, we have shown that Lin and Sontag's bounded control law for disturbance-free systems also ensures Strong iISS provided that a condition on the 
growth rate of the input term is satisfied. We have also shown that iISS can be obtained under relaxed conditions. We have illustrated the applicability of our results on a spacecraft velocity control example. Focusing on the specific class of LTI systems with skew-symmetric matrix, we have shown that a simple linear static state-feedback ensures Strong iISS. This result was illustrated on an academic example, that underlined the limits of the input threshold estimate and suggests that further work is needed to choose the feedback gains in order to obtain a tighter input threshold estimate. Finally, further research can be envisioned to check the robustness properties of saturated stabilization of chains of integrators.

\section{References}

Angeli, D., Chitour, Y., Marconi, L., Jan. 2005. Robust stabilization via saturated feedback. IEEE Trans. on Automat. Contr. 50 (12), 1997-2014.

Angeli, D., Sontag, E., 1999. Forward completeness, unboundedness observability, and their Lyapunov characterizations. Syst. \& Contr. Letters 38, 209-217.

Angeli, D., Sontag, E., Wang, Y., 2000. A characterization of integral input to state stability. IEEE Trans. on Automat. Contr. 45, 1082-1097.

Arcak, M., Teel, A., 2002. Input-to-state stability for a class of Lurie systems. Automatica 38 (11), $1945-1949$.

Chaillet, A., Angeli, D., Ito, H., Sept 2014a. Combining iISS and ISS with respect to small inputs: the Strong iISS property. IEEE Trans. on Automat. Contr. 59 (9), 2518-2524.

Chaillet, A., Angeli, D., Ito, H., 2014b. Strong iISS is preserved under cascade interconnection. Provisionnally accepted at Automatica.

Freeman, R., Kokotović, P., 1996. Robust Nonlinear control design: State-space and Lyapunov control techniques. Birkhäuser, Boston.

Fuller, A., 1969. In-the-large stability of relay and saturating control systems with linear controllers. International Journal of Control 10 (4), 457-480.

Khalil, H., 2002. Nonlinear systems. Prentice Hall, 3rd ed., New York.

Krstic, M., Li, Z.-H., 1998. Inverse optimal design of Input-to-State Stabilizing nonlinear controllers. IEEE Trans. on Automat. Contr. 43 (3), 336-350.

Liberzon, D., 2002. ISS and integral-ISS disturbance attenuation with bounded controls. Proc. 41st. IEEE Conf. Decision Contr. 3, 2501-2506.

Liberzon, D., Sontag, E., Wang, Y., 2002. Universal construction of feedback laws achieving ISS and integral-ISS disturbance attenuation. Syst. \& Contr. Letters 46, 111-127.

Lin, Y., Sontag, E., 1991. A universal formula for stabilization with bounded controls. Syst. \& Contr. Letters 16 (6), 393-397.

Liu, W., Chitour, Y., Sontag, E., 1996. On finite-gain stabilizability of linear systems subject to input saturation. SIAM Journal on Control and Optimization 34 (4), 1190-1219.

Malisoff, M., Rifford, L., Sontag, E., 2004. Global asymptotic controllability implies Input-to-State stabilization. SIAM J. Control Optim. 42, 2221-2238.

Mazenc, F., Praly, L., 1996. Adding integrators, saturated controls and global asymptotic stabilization of feedforward systems. IEEE Trans. on Automat. Contr. 41 (11), 1559-1579.

Megretski, A., 1996. $l_{2}$ bibo output feedback stabilization with saturated control. In: In IFAC World Congress. pp. 435-440.

Saberi, A., Hou, P., Stoorvogel, A., 2000. On simultaneous global external and global internal stabilization of critically unstable linear systems with saturating actuators. IEEE Trans. on Automat. Contr. 45 (6), 368-378.

Sontag, E., 1989a. Smooth stabilization implies coprime factorization. IEEE Trans. on Automat. Contr. 34 (4), $435-443$.

Sontag, E., 1989b. A "universal" construction of Artstein's theorem on nonlinear stabilization. Syst. \& Contr. Letters 13 (2), 117-123.

Sontag, E., 1990. Further facts about Input-to-State stabilization. IEEE Trans. on Automat. Contr. 35, $473-476$.

Sontag, E., 1998a. Comments on integral variants of ISS. Syst. \& Contr. Letters 34, 93-100.

Sontag, E., 1998b. Mathematical Control Theory: Deterministic Finite Dimensional Systems. Springer Verlag, New York.

Sontag, E., 2006. Input to state stability: Basic concepts and results. Lecture Notes in Mathematics. Springer-Verlag, Berlin, Ch. in Nonlinear and Optimal Control Theory, pp. 163-220, p. Nistri and G. Stefani eds.

Sontag, E., Sussmann, H., 1990. Nonlinear output feedback design for linear systems with saturating controls. In: Proc. 29th. IEEE Conf. Decision Contr. Honolulu, HI, pp. 3414-3416.

Sontag, E., Wang, Y., 1995. On characterizations of the Input-to-State Stability property. Syst. \& Contr. Letters $24,351-359$ 
Sussmann, H., Sontag, E., Yang, Y., 1993. A general result on the stabilization of linear systems using bounded controls. Proc. of the 32nd IEEE Conference on Decision and Control, 1802-1807.

Sussmann, H., Sontag, E., Yang, Y., 1994. A general result on the stabilization of linear systems using bounded controls. IEEE Trans. on Automat. Contr. 39 (12), 2411-2425.

Sussmann, H., Yang, Y., December 1991. On the stabilizability of multiple integrators by means of bounded feedback controls. In: Proc. 30th. IEEE Conf. Decision Contr. Brighton, UK.

Teel, A., 1992. Global stabilization and restricted tracking for multiple integrators with bounded controls. Syst. \& Contr. Letters 18 (3), 165-171.

Teel, A., 1996. A nonlinear small gain theorem for the analysis of control systems with saturation. IEEE Trans. on Automat. Contr. 40, 1256-1270.

Teel, A., Praly, L., 2000. On assigning the derivative of a disturbance attenuation Control Lyapunov Function. Math. of Cont., Sign. and Syst. 13, 95-124.

Wang, X., Saberi, A., Stoorvogel, A., Grip, H., 2012. Control of a chain of integrators subject to actuator saturation and disturbances. International Journal of Robust and Nonlinear Control 22 (14), 1562-1570. 